\title{
Direct quantification of single red blood cell hemoglobin concentration with multiphoton microscopy
}

\author{
Andrew T. Francis ${ }^{1}$, Melanie J. Shears ${ }^{2}$, Sean C. Murphy², Dan Fu' ${ }^{1 *}$ \\ ${ }^{1}$ Department of Chemistry, University of Washington, Seattle, WA 98195, United States \\ ${ }^{2}$ Department of Laboratory Medicine and Center for Emerging and Re-emerging Infectious Diseases, University of Washington, 750 Republican St., \\ Seattle, WA 98109, United States
}

S1. Energy diagrams of SRS, TPA, and ESA

S2. Wavelength survey of signal/transient lifetime at size probe wavelengths

S3. High resolution SRS spectra of $\mathrm{H}_{2} \mathrm{O}, 1 \mathrm{X}$ PBS, $\mathrm{H}_{2} \mathrm{O}(\mathrm{pH} 8), 10 \%$ (w/v) BSA, 20\% (w/v) glucose

S4. Transform limited SRS spectra of $\mathrm{H}_{2} \mathrm{O}, 10 \%$ BSA, and oleic acid

S5. Giemsa-stain thin blood smears for parasitemia validation 
Derivation of ratiometric fitting equation (Equation 3) from Equations 1 and 2:

$$
\begin{aligned}
& S_{1}=\left[H_{2} O\right] \times \sigma_{H_{2} O}+[H b] \times \sigma_{1, H b} \\
& S_{2}=[H b] \times \sigma_{2, H b}
\end{aligned}
$$

The ratio of the two gives

$$
\begin{gathered}
\frac{S_{2}}{S_{1}}=\frac{[H b] \times \sigma_{2, H b}}{[H b] \times \sigma_{1, H b}+\left[H_{2} O\right] \times \sigma_{H_{2} O}} \\
\frac{S_{2}}{S_{1}}=\frac{[H b] \times \sigma_{2, H b}+\frac{\sigma_{2, H b}}{\sigma_{1, H b}}\left(\left[H_{2} O\right] \times \sigma_{H_{2} O}\right)-\frac{\sigma_{2, H b}}{\sigma_{1, H b}}\left(\left[H_{2} O\right] \times \sigma_{H_{2} O}\right)}{[H b] \times \sigma_{1, H b}+\left[H_{2} O\right] \times \sigma_{H_{2} O}} \\
\frac{S_{2}}{S_{1}}=\frac{\frac{\sigma_{2, H b}}{\sigma_{1, H b}}\left([H b] \times \sigma_{1, H b}+\left[H_{2} O\right] \times \sigma_{H_{2} O}\right)-\frac{\sigma_{2, H b}}{\sigma_{1, H b}}\left(\left[H_{2} O\right] \times \sigma_{H_{2} O}\right)}{[H b] \times \sigma_{1, H b}+\left[H_{2} O\right] \times \sigma_{H_{2} O}} \\
\frac{S_{2}}{S_{1}}=\frac{\sigma_{2, H b}}{\sigma_{1, H b}}-\frac{\frac{\sigma_{2, H b}}{\sigma_{1, H b}}\left(\left[H_{2} O\right] \times \sigma_{H_{2} O}\right)}{\left[S_{1}\right.}=\frac{\sigma_{2, H b}}{\sigma_{1, H b}}-\frac{1}{[H b] \times \sigma_{1, H b}+\left[H_{2} O\right] \times \sigma_{H_{2} O}} \\
\frac{\sigma_{1, H b}}{\sigma_{2, H b}\left(\left[H_{2} O\right] \times \sigma_{H_{2} O} O\right.}\left([H b] \times \sigma_{1, H b}+\left[H_{2} O\right] \times \sigma_{H_{2} O}\right) \\
A=\frac{\sigma_{2, H b}}{\sigma_{1, H b}} \quad B=-\frac{\sigma_{1, H b}^{2}}{\sigma_{2, H b}\left(\left[H_{2} O\right] \times \sigma_{H_{2} O} O\right.} \quad C=\frac{\sigma_{1, H b}}{\sigma_{2, H b}}
\end{gathered}
$$




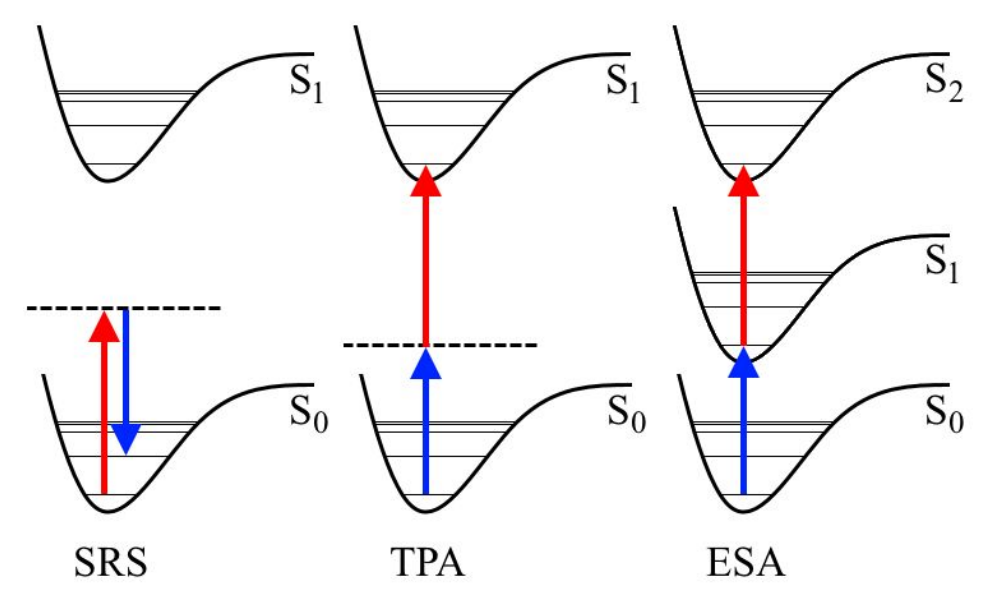

Figure S1. Energy diagrams of stimulated Raman scattering (SRS), two-photon absorption (TPA), and excited state absorption (ESA). The wavelengths used were $1040 \mathrm{~nm}$ (blue) and $765 \mathrm{~nm}$ (red).
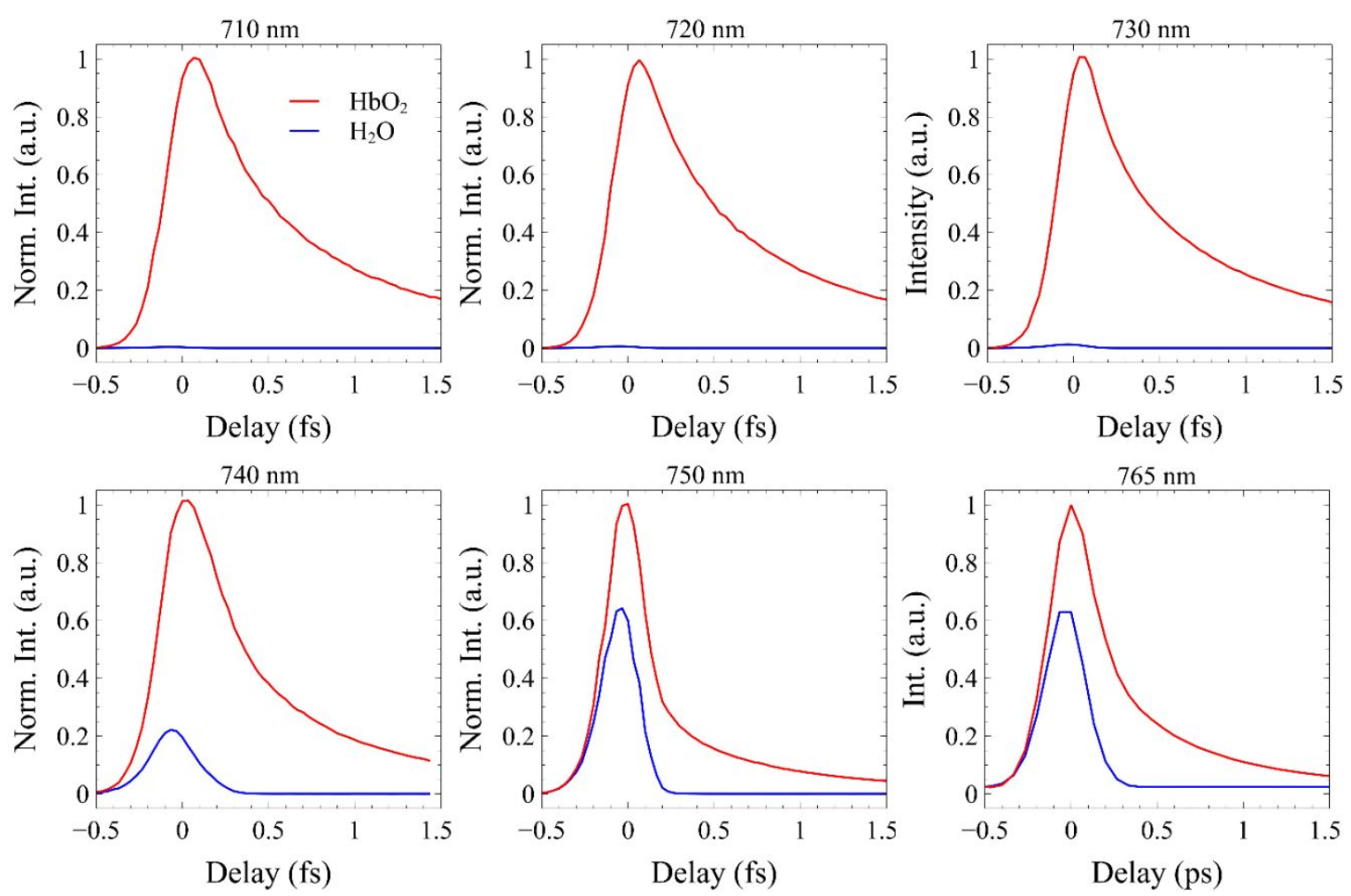

Figure S2. Wavelength survey for simultaneous excitation of hemoglobin $\left(\mathrm{HbO}_{2}\right)$ with TAM and water $\left(\mathrm{H}_{2} \mathrm{O}\right)$ with $\mathrm{SRS}$. Pump wavelength was $1040 \mathrm{~nm}$ for all traces shown. 

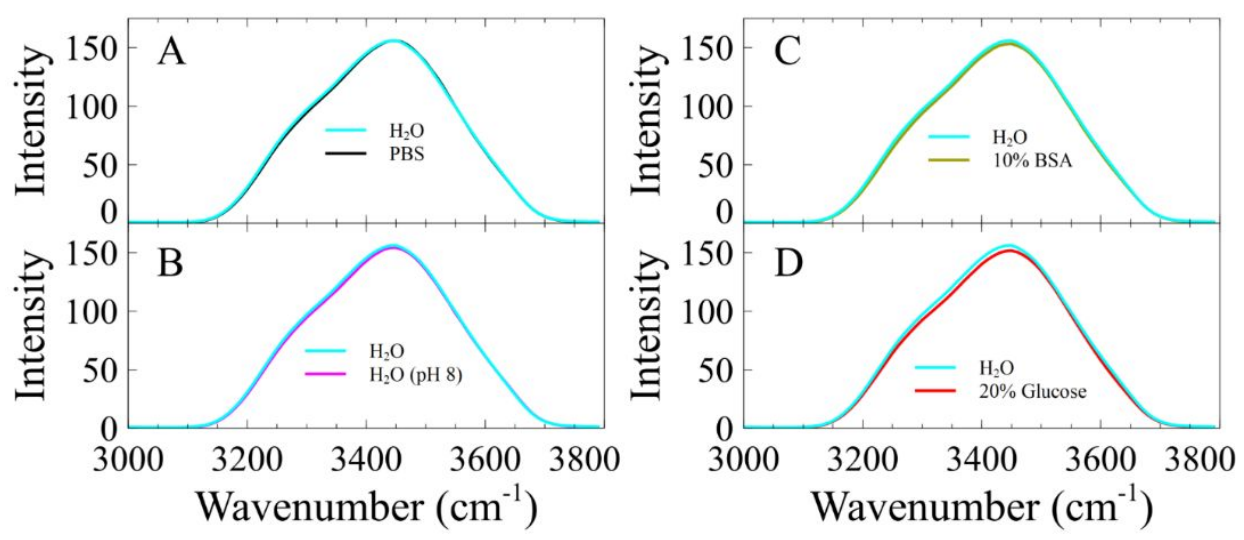

Figure S3. High resolution SRS spectra of four common cellular constituents in water. Comparisons of the $\mathrm{OH}$ stretching mode between water and A) 1X PBS, B) water (pH8), C) 10\% BSA, and D) 20\% glucose.

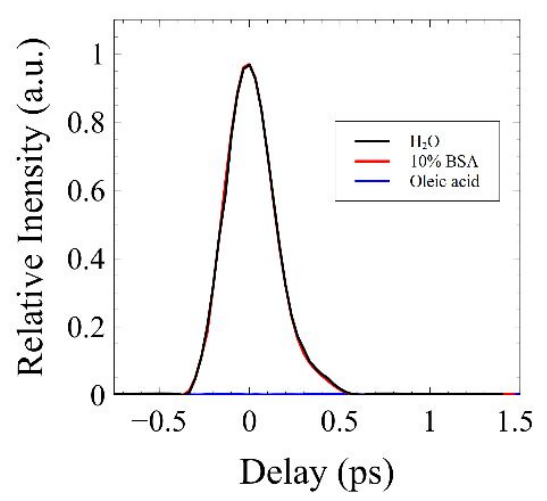

Figure S4. SRS spectra of water, 10\% BSA and oleic acid excited at $765 \mathrm{~nm} / 1040 \mathrm{~nm}$. 


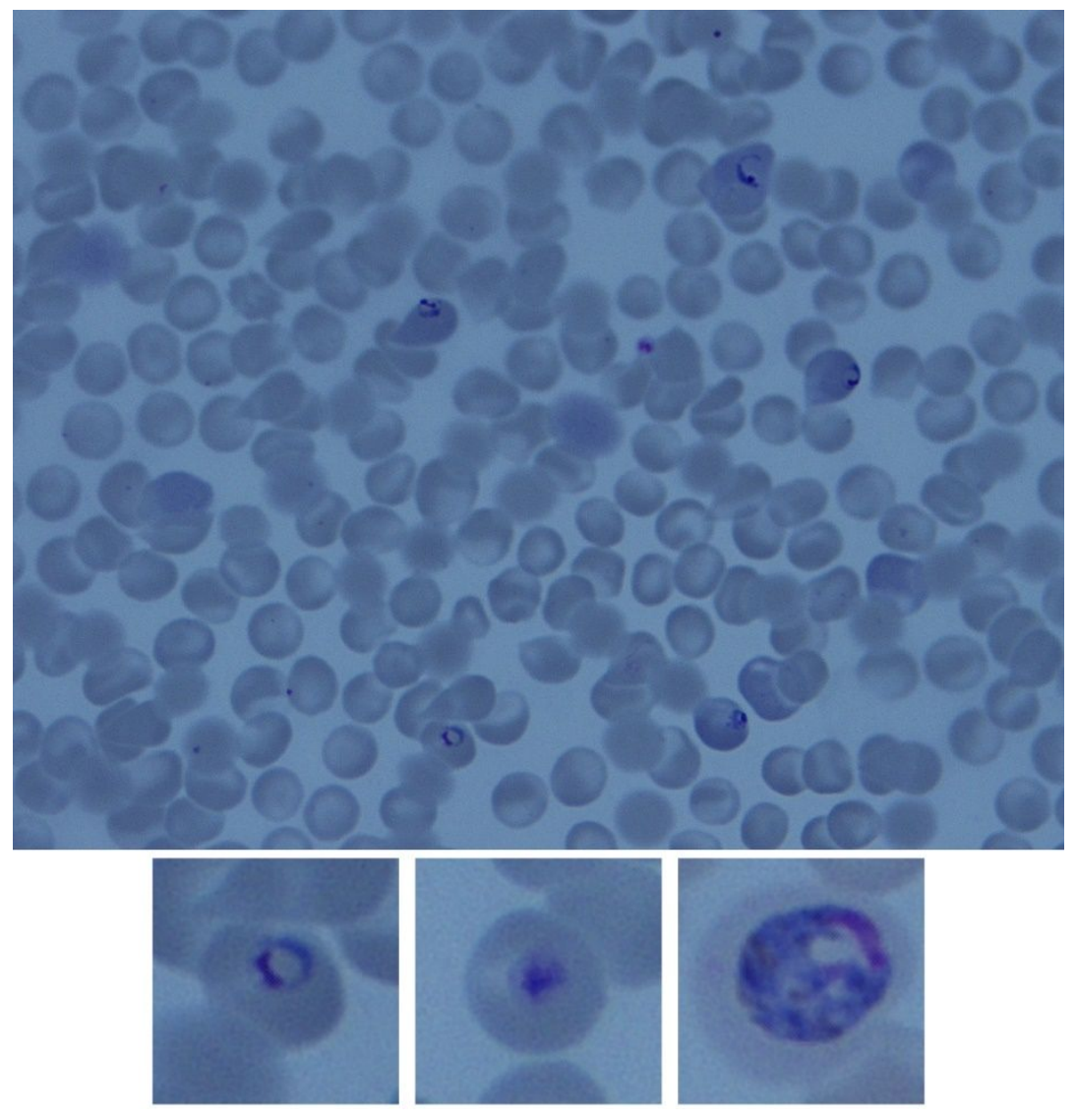

Figure S5. Giemsa-stained thin blood smears. 